especially in regard to the different cell types, which can produce IL-10. Here the authors analysed whether IL-10 producing CD4 T cells have a beneficial effect in murine lupus.

Materials and methods Lupus-prone NZB/W F1 mice were repeatedly high-dose tolerised with $\mathrm{SmD1}(83-119)$ and analysed for SmD1(830119)-specific CD4 cells producing IL-10. Function of IL-10 on autoantibody production was analysed in an in vitro assay based on ELISA. Young, healthy NZB/W F1 mice were preventively treated with whole CD4 $\mathrm{T}$ cells from SmD1(83-119)- tolerised NZB/W F1 mice. And finally old, diseased NZB/W F1 were treated with polyclonal CD4 IL-10+ T cells generated in vitro.

Results First, the authors detected SmD1(83-119)-specific CD4 IL-10+ T cells after their previously published SmD1(83119) high dose tolerisation protocol and the authors were able to increase the number of these cells by an additional booster immunisation with SmD1(83-119), second, the authors showed that IL-10 has a suppressive effect on anti-dsDNA autoantibody production at least in vitro, third, the authors achieved a temporary beneficial effect on proteinuria course and autoantibody development by transfer of CD4 T cells containing up to $0.04 \% \mathrm{SmD} 1(83-119)$-specific CD4 IL-10+ T cells from SmD1(83-119) high dose tolerised NZB/W F1 mice into untreated NZB/W F1 mice, and fourth, the authors were able to treat full blown lupus disease in old female NZB/W F1 mice with in vitro generated polyclonal CD4 IL-10+ T cells improving significantly the survival of these mice in contrast to a control transfer of in vitro generated polyclonal CD4 interferon $\gamma+$ $T$ cells or phosphate-buffered saline treated control mice.

Conclusions The authors conclude that IL-10 produced by CD4 $\mathrm{T}$ cells has a beneficial effect in murine lupus, even in full-blown disease in old, severely ill lupus-prone NZB/W F1 mice. Therefore, treatment of lupus prone mice with this cell type is an interesting new approach.

\section{A171 CD4 T CELLS PRODUCING IL-10 HAVE A BENEFICIAL EFFECT IN MURINE LUPUS}

R Undeutsch,1,2 J Y Humrich,1,2 A Papendieck,1,2 G Riemekasten' 1Department of Rheumatology and Clinical Immunology, Charité - University Medicine, Berlin, Germany; ${ }^{2}$ German Rheumatology Research Centre, a Leibniz Institute, Berlin, Germany

\subsection{6/ard.2010.149013.14}

Background and objectives Systemic lupus erythematosus is a severe systemic chronic autoimmune disease and New Zealand black $\times$ New Zealand white $(\mathrm{NZB} / \mathrm{W})$ F1 mice are commonly used to study this disease. The role of interleukin 10 (IL-10) in lupus is controversial and needs to be clarified, 